\title{
BMJ Open Scoping review of physical rehabilitation interventions in long-term care: protocol for tools, models of delivery, outcomes and quality indicators
}

\author{
Caitlin McArthur, ${ }^{1}$ Jenna Gibbs, ${ }^{1}$ Alexandra Papaioannou, ${ }^{2}$ John Hirdes, ${ }^{3}$ \\ James Milligan, ${ }^{4,5,6}$ Katherine Berg, ${ }^{7}$ Lora Giangregorio ${ }^{3,8,9}$
}

To cite: McArthur C, Gibbs J, Papaioannou A, et al. Scoping review of physical rehabilitation interventions in long-term care: protocol for tools, models of delivery, outcomes and quality indicators. BMJ Open 2015;5: e007528. doi:10.1136/ bmjopen-2014-007528

- Prepublication history and additional material is available. To view please visit the journal (http://dx.doi.org/ 10.1136/bmjopen-2014007528).

Received 22 December 2014 Revised 6 May 2015 Accepted 7 May 2015

CrossMark

For numbered affiliations see end of article.

Correspondence to

Caitlin McArthur;

cmcarthu@uwaterloo.ca

\section{ABSTRACT}

Introduction: A growing number of medically complex older adults reside in long-term care (LTC) and often require physical rehabilitation (PR). While PR is effective at maintaining or improving a patient's physical function, the breadth of PR interventions evaluated in LTC, which outcomes or quality indicators (QI) can be used to evaluate PR, and what tools or models can be used to determine eligibility for PR services remain unknown.

Methods and analysis: A scoping review will be conducted to address the following research questions: (1) What types of PR have been evaluated for efficacy or effectiveness in LTC? (2) Which outcomes or Qls have been used when evaluating PR interventions in LTC, and how can this inform evaluation of PR using existing QIs in the Canadian context? (3) What tools or models exist or have been validated for decision-making in the allocation of PR resources in LTC? We will conduct a comprehensive literature search in MEDLINE, EMBASE, CINAHL, Cochrane Database of Systematic Reviews, Physiotherapy Evidence Database (PEDro) and Occupational Therapy Systematic Evaluation of Evidence database (OTseeker) and a structured grey literature search. Two team members will screen articles and abstract the data. The results will be displayed according to the research question they address. Data abstracted regarding outcomes and QIs will be mapped onto existing, publicly reported QIs used in Ontario, Canada.

Ethics and dissemination: The scoping review will synthesise the characteristics of PR interventions described in the literature, the outcomes used to evaluate them and tools to determine eligibility for services. The review will be the first step in formally identifying what outcomes and QIs have been used to evaluate PR in LTC, and will be used to inform a stakeholder consensus process exploring the same question. The scoping review may also identify knowledge gaps. The results will be disseminated via publication and presentation at conferences, in addition to a 1-day stakeholder meeting.
Strengths and limitations of this study

A strength of this study is that it will be the first review using both peer reviewed and grey literature to synthesise what is known about interventions, outcomes, quality indicators (QIs) and tools regarding decision-making for physical rehabilitation (PR) in long-term care (LTC).

- The scoping review will be the first step in a process to determine which outcomes and QIs could be used to evaluate PR in LTC in the Canadian context.

- A limitation of scoping reviews is that they inherently provide breadth and not depth about a topic; however, this study will provide a breadth of knowledge about active and passive PR, where a depth of knowledge has already been considered via systematic reviews. Additionally, the results may be applicable to many disciplines in LTC-including physical therapy, occupational therapy and nursing, in addition to administration and policymakers.

- A limitation of this study is that only studies and grey literature published in English will be included, which will limit the scope of this review to articles published in English speaking countries or to those that have funds for translation services.

- A limitation of the proposed scoping review is that recommendations for rehabilitation approaches for all international groups will not be possible as it may be found in different literature not included in the search strategy. For example, the term intermediate care is used in the UK for short stays of rehabilitation in nursing homes and might not be captured in our search. By using a standard definition of LTC in our inclusion and exclusion criteria, we have attempted to capture as much as the international literature as possible.

\section{INTRODUCTION}

Currently, $7.1 \%$ of all older adults in Canada live in long-term care (LTC) and it has been estimated that by the year 2036, the number 
of individuals living in institutional care facilities will be more than double. ${ }^{1}{ }^{2}$ Residents in LTC often have combinations of threats to well-being, including pain, disability and mental health issues. ${ }^{3}$ Therefore, they often require support by a multidisciplinary team, including those providing physical rehabilitation (PR). ${ }^{34}$

PR is defined by The Canadian Physiotherapy Association as:

Promoting optimal mobility, physical activity and overall health and wellness; Preventing disease, injury, and disability; Managing acute and chronic conditions, activity limitations, and participation restrictions; Improving and maintaining optimal functional independence and physical performance; Rehabilitating injury and the effects of disease or disability with therapeutic exercise programs and other interventions; and Educating and planning maintenance and support programs to prevent re-occurrence, re-injury or functional decline. (http:// www.physiotherapy.ca/getmedia/e3f53048-d8e0-416b-9c9d38277c0e6643/DoPEN(final).pdf.aspx $)^{5}$

PR can therefore be active by involving physical exertion by the resident (eg, participating in exercise) or passive via external stimulation (eg, therapeutic modalities such as ultrasound or whole body vibration). PR can be delivered in a group setting or on an individual basis by a physiotherapist, kinesiologist, fitness instructor, nurse or other staff involved in the LTC home.

The goal of PR to maintain and improve mobility, physical activity, and overall health and wellness is clear; however, identifying interventions and models of delivery for residents in LTC remain to be determined. ${ }^{5}{ }^{6} \mathrm{~A}$ systematic review of active PR in LTC concluded that there is a lack of evidence in this area and revealed heterogeneity in the goals of interventions as well as in the time allocated to PR, the staff delivering PR, and the model of delivery of PR. ${ }^{6}$ For example, some PR interventions focus on general strength and balance and are delivered by a PR assistant in a group format for $45 \mathrm{~min}$ three times per week, while others focus on specific activities of daily living and are delivered by a restorative care nurse on an individual basis for $1 \mathrm{~h}$ daily. ${ }^{6}$ Additionally, active and passive PR techniques have not been considered to date in one review.

In addition to heterogeneity surrounding PR interventions and which healthcare member delivers PR care, the outcomes used to evaluate PR are not consistent. A plethora of constructs and outcome measures have been used to evaluate the effect of PR at the resident level; yet constructs measured to evaluate PR at the facility or system levels are limited. Constructs measured to evaluate PR at the resident level include: activities of daily living, balance, muscle power, flexibility, exercise tolerance, physical activity, mood, cognitive performance, quality of life, fear of falling and perceived health status. ${ }^{6}$ While resident-level evaluation is useful for PR treatment planning and evaluation, facility-level and system-level evaluation can allow for comparison of outcomes within and across LTC homes and provide support for quality improvement strategies. Indeed, it has been suggested that measurement of outcomes, processes and structures at multiple levels of the healthcare system are required to facilitate improvement. ${ }^{78}$

Quality indicators (QI) are used to monitor and improve quality of care in LTC at the facility and system level. ${ }^{9}{ }^{10}$ A QI is defined as resident-level data aggregated at the facility level and expressed as fractions, where the numerator reflects the number of residents with a particular outcome and the denominator reflects the number of residents at risk for developing an outcome, who are not otherwise excluded from the QI. ${ }^{9}$ In Ontario, 12 QIs are publically reported for each LTC facility through Health Quality Ontario including: wait times, incontinence, activities of daily living, effective cognitive function, pain, emergency department visits, falls, pressure ulcers, restraints, medication safety, human health resources and infections. ${ }^{11}$ Existing QIs have the potential to reflect the quality of rehabilitative care at the facility level within and across LTC homes. Thus, there is a need to determine which would best reflect an evaluation of PR.

Uncertainty surrounding interventions, delivery and evaluation of outcomes of PR in LTC is compounded by additional uncertainty regarding which residents in LTC might benefit from PR services in LTC. To date, there are jurisdictional differences in utilisation rates for PR across Canada and internationally. ${ }^{3} 1213$ Certain studies reveal that older residents with cognitive impairment are less likely to receive PR services ${ }^{11}$ despite evidence supporting the efficacy of PR for improving function for individuals with cognitive impairment. ${ }^{14-16}$ To add to the complexity of who might benefit from PR in LTC, there are different categories of residents in LTC such as those admitted for short stays with a definite number of days to rehabilitate after an acute event and return to the community compared with those admitted for long stays requiring ongoing care. ${ }^{17}$ While the majority of residents in LTC in Canada are in long-stay programmes such that they require residence in LTC indefinitely, there has recently been an increase in the number of short-stay programmes in LTC in Ontario. ${ }^{18}$ There is also international variation regarding the goals and length of stay for residents in LTC. For example, in the USA, residents admitted to skilled nursing facilities often have the goal of returning home, while there are wards dedicated to PR in European LTC homes. ${ }^{13} 19$ The length of stay and goals for PR must be considered in goal setting, delivery and allocation of rehabilitative care.

The aim of the scoping review is to synthesise evidence regarding which active and passive PR interventions and models of delivery have been evaluated, what outcomes and QIs have been used to evaluate them and tools or models used to determine eligibility for services. The synthesis will identify the scope of PR interventions and how they have been evaluated; this can be used to 
inform future research and policy making. A scoping review has been chosen to provide breadth on the topic, rather than depth, and to include a variety of publication types including grey literature (eg, policy papers, reports and clinical practice guidelines). ${ }^{20}$

The objectives of the scoping review are to:

1. Characterise the types of active and passive PR interventions (eg, therapeutic goal, frequency, mode of delivery) that have been evaluated for efficacy/effectiveness in LTC;

2. Identify which outcomes at the person, facility or system levels have been used when evaluating the efficacy/effectiveness of PR interventions in LTC;

3. Map the identified outcomes used when evaluating the efficacy/effectiveness of PR in LTC to the existing QIs in LTC across Canada so as to inform future programme design and implementation;

4. Characterise any tools or models that exist or have been validated for decision-making in the allocation of PR resources in LTC;

5. Use the available evidence and stakeholder consultation to identify which existing or new outcomes and QIs could be used to evaluate PR at the person, facility or system levels.

\section{METHODS AND ANALYSIS}

We will use the framework proposed by Arksey and $\mathrm{O}^{\prime}$ Malley, ${ }^{20}$ and the suggestions proposed by Levac et $a l^{21}$ to guide the scoping review methodology.

\section{Research questions}

The team defined the concepts, target population and outcomes of interest, and has drafted three main research questions for the scoping review. The main concept of interest is PR in LTC. The Canadian Physiotherapy Association's Description of Physiotherapy in Canada, 2012, will be used to define PR (see introduction). (http://www.physiotherapy.ca/getmedia/e3f53048d8e0-416b-9c9d-38277c0e6643/DoPEN(final) .pdf.aspx $)^{5}$
The population of interest includes adults aged 65 years or older residing in a LTC facility. LTC will be defined as a home for residents who are unable to live independently, requiring access to nursing, personal care, support and/or supervision. ${ }^{22}$ The research questions and outcomes of interest are outlined in table 1 .

\section{Identifying relevant studies}

\section{Licensed journal databases}

Systematic searches for peer-reviewed articles will be conducted in the following licensed databases: MEDLINE PubMed (1946-present), EMBASE Ovid (1974-present), CINAHL (1981-present), Cochrane Database of Systematic Reviews (1994-present), the Physiotherapy Evidence Database (PEDro), and the Occupational Therapy Systematic Evaluation of Evidence database (OTseeker). The searches will be limited to papers with full texts in English. The search strategies will use text and indexing terms to capture the key concepts: LTC, PR, interventions that have been evaluated, elderly, decisions regarding resource allocation, tools to assist in decision-making and evaluation including QIs (see online supplementary file 1, eg, of the search filters run in MEDLINE). Concepts will be combined using the Boolean Operator AND, and the search terms within each concept will be combined with OR. Keywords will be searched using truncation and phrase symbols when appropriate to ensure precise and comprehensive results. Results from one research question's search results may be applicable to a different question; therefore, the results from the both searches will be combined (using the Boolean Operator OR) and there will be one final search for each database.

\section{Grey literature}

A grey literature search will be conducted to find clinical trials in progress, practice guidelines not indexed in databases, policy, government reports, legislation and

Table 1 Research question and outcomes of interest

\begin{tabular}{ll}
\hline Research question & Outcomes of interest \\
\hline 1. What types of PR have been evaluated for efficacy or & Types of PR interventions including: \\
effectiveness in LTC? & Therapeutic goals (eg, improve strength, decrease falls) \\
& Modes of delivery (eg, delegated care, direct care) \\
2. Which outcomes or quality indicators have been used when & Outcomes or quality indicators* used at the: \\
evaluating the efficacy or effectiveness of PR interventions & Resident level (eg, ADL functioning) \\
in LTC? & Facility level (eg, monthly falls rate) \\
3. What tools or models exist or have been validated for & Tools or models for allocation of resources (eg, criteria or \\
decision-making in the allocation of PR resources in LTC? & algorithms for determining who receives services) \\
\hline${ }^{*}$ Quality indicators will be defined as person-level data aggregated at the facility level and expressed as fractions, where the numerator \\
reflects the number of residents with a particular outcome and the denominator reflects the number of residents at risk for developing an \\
outcome who are not otherwise excluded from the QI. ${ }^{9}$. \\
ADL, activities of daily living; LTC, long-term care; PR, physical rehabilitation; QI, quality indicators.
\end{tabular}


conference papers using the following websites: Canadian Institute for Health Information, Ministry of Health and LTC, National Institute of Health, and the Government and Legislative Libraries Online Publications Portal, Canadian Physiotherapy Association, Ontario Long-term Care Association, American Academy of Physical Medicine and Rehabilitation and the University of Waterloo's library catalogue (a full government depository library). We will also do a broad Google search, focusing on the first few pages of relevancy ranked results, until the results become mostly irrelevant. The websites, search engine and catalogue will be searched for documents using selected, common keywords from the online supplementary appendix A and then the found documents will be searched for appropriate keywords to identify relevant information.

\section{Hand searching}

We will hand search reference lists of included articles for more relevant citations, and we will identify and contact researchers or opinion leaders in the area of PR in LTC.

\section{Selection of studies for inclusion}

Two team members (CM and one other) will review the title, abstract and descriptors of identified citations and apply the inclusion and exclusion criteria discussed below. If there is not enough information to make an informed decision, the article will be retrieved. Two team members will then independently assess all full-text studies/reports for inclusion by applying the inclusion and exclusion criteria again. Disagreement will be resolved through discussion or third party adjudication. For the grey literature search, the two team members will apply the inclusion and exclusion criteria to the citations gathered from hand searching and grey literature searches as those citations are found. The searches for, and selection of, these documents will happen concurrently. The screening process will be pilot tested on 5\% of both papers obtained from licensed databases and the grey literature search. The screening forms will be modified if there is low agreement between the two team members that is indicated by a $\kappa$ statistic less than 0.5 .

\section{Inclusion criteria}

The following research and study designs will be considered: case studies, mixed methods, prospective, longitudinal, retrospective case-control, randomised controlled trials, quasi-randomised clinical trials or controlled clinical trials, clinical practice guidelines, systematic reviews and relevant reports generated by policymakers. For a study to be included, more than half of the participants will have to be elderly, defined as individuals with a median or mean age of $\geq 65$ years, and residing in an LTC facility defined as a home for residents who are unable to live independently, requiring access to nursing, personal care, support and/or supervision. If a study has participants from multiple populations (eg, multiple ages or settings), results relating to the population of interest (ie, residents $\geq 65$ years of age residing in LTC) must be presented separately for the study to be included. Included papers must focus on PR as defined by the Canadian Physiotherapy Association. To be included, studies or documents must focus on either a PR intervention, a tool, model or framework for systemlevel decision-making regarding eligibility for PR services, or describe, evaluate or provide evidence for a QI that could be used to evaluate PR. Exclusion criteria: Papers that discuss tools or models that have not been validated will be excluded. For a tool or model to be considered validated proof of face, construct or criterion validity must be demonstrated. In addition, non-English full-text papers, clinical commentaries, editorials, interviews, lectures, legal cases, letters, newspaper articles, patient education handout, abstracts or unpublished literature will be excluded.

\section{Charting the data}

A data abstraction form was compiled with input from the research team. The data abstraction form will be pilot tested on a random sample of 10 articles and revised as needed. Two team members will then use the pretested data abstraction form to abstract data from included studies, tools or models. Disagreement will be resolved by consensus or third party adjudication. Charting will be an iterative process with the research team continually updating the data abstraction form. The data that will be abstracted as described in table 2 .

\section{Summarising and reporting the findings}

We will display information sources according to the research question addressed.

\section{Summary}

Infographics, such as bar graphs and maps, will be used to visually display year of publication, country of origin, proportion of articles involving short-stay and long-stay residents, and the proportion of articles that address each research question.

Research question 1: interventions-Interventions will be sorted and presented based on the QI they address (see below). Under each QI the interventions will then be grouped based on the level of intervention delivery (person, facility or system) and a summary of the level of evidence based on the study design ${ }^{21}$ will be presented. Under each level of intervention a description of the type, frequency, intensity, time/volume and person delivering the intervention will be presented in table format. The types of PR intervention will be grouped based on the target of each intervention, for example, interventions will be grouped based on those targeting strength, balance, aerobic endurance, functional skills training or those that are 'multi-target', involving at least two of the previously mentioned targets. 
Table 2 Data abstraction for the three research questions

\section{Data to be abstracted}

\section{Summary}

Research question 1: interventions

Research question 2: outcomes and quality indicators

Research question 3: tools, models or frameworks for decision-making
1. Title of the study

2. Authors of the study

3. Location of study (country)

4. Which research question does the study address? (1,2 and/or 3$)$

5. Type of literature (published peer-reviewed article, report, policy paper)

6. Length of stay of residents (short or long): as defined by the Ontario Ministry of Health and Long-term Care, long-stay residents are those residing in the home for more than 90 days ${ }^{15}$

7. Description of participants (age, sex, cognition, inclusion/exclusion criteria)

8. Description of facility or unit type (eg, specialised nursing facility, nursing home, long-term care facility)

1. Study design

- Description of study design, eg, randomised controlled trial, systematic review, etc)

- Level of evidence I-VI, based on study design (from evidence-based practice in nursing healthcare: a guide to best practice ${ }^{23}$ )

2. Description of intervention:

- Therapeutic goals

- Frequency

- Intensity

- Time/volume

- Duration

- Type (eg, strength, balance, multicomponent)

- Who delivered the intervention

- Level of intervention delivery (person, facility, system)

- Which QI(s) is/are addressed

A. Outcomes from trials:

1. Outcome of interest: construct(s) measured, outcome measures used

2. Outcome level: person, facility or system

B. Quality indicators:

1. Name of quality indicator

2. Description of quality indicator

3. Description of calculation of quality indicator (numerator, denominator, risk adjustment, inclusion/exclusion criteria)

4. Evidence to support the use of the quality indicator:

- Description of data source for derivation of QI

- Stakeholder engagement process (yes/no, description of process)

- Average prevalence/incidence, variance

- Sensitivity to change, timeframe for improvement

1. Name of tool, model or framework

2. Description of tool, model or framework

3. Population tool, model or framework used with (eg, short or long stay)

4. Country of implementation

5. Description of validation or implementation process for tool, model or framework

QI, quality indicators.

Research question 2: outcomes and OIs-The frequency of the constructs and outcome measures used to measure the constructs will be tallied. Interventions will be mapped onto the QI they address by listing the articles by the outcomes measured. For example, if a study measures falls as an outcome, that article would be listed under the QI 'falls'. The articles will be listed under the domains of the existing QIs in Ontario (ie, wait times, incontinence, activities of daily living, effective cognitive function, pain, emergency department visits, falls, pressure ulcers, restraints, medication safety, human health resources and infections). If an outcome is reported that does not address one of the current QIs, it will be identified and articles reporting this outcome will be listed under the domain of the outcome.

Research question 3: tools, models or frameworks for decisionmaking - A description of the identified tools, models or frameworks for decision-making, the population it has 
been used with, the country of implementation, and the validation and implementation processes will be displayed and compared in a table.

\section{Consultation with stakeholders}

We have identified four groups of stakeholders: policymakers, rehabilitation professionals, administrators and healthcare providers in LTC and residents and families. During each stage, we will consult the stakeholders via videoconference or teleconference or through surveys or one-on-one consultations so as to identify questions important for decision-making, to get input on key messages and definitions of terms or QIs for PR in the LTC sector and to identify areas for future research. Additionally, we will hold a 1-day stakeholder meeting on completion of the review that will bring together key stakeholders across multiple disciplines, including policymakers and knowledge users across PR, occupational therapy, nursing and kinesiology within the LTC sector. The stakeholder meeting will serve three purposes: (1) to disseminate the results of the scoping review to key stakeholders, (2) to engage in a formal consensus process using nominal group technique ${ }^{24} 25$ to determine which new or existing outcomes and QIs could be used to evaluate PR in LTC and (3) to identify any additional emerging issues and future research priorities for PR in LTC. The scoping review fits into a larger, evolving programme of research focused on improving delivery and evaluation of PR in LTC.

\section{ETHICS AND DISSEMINATION}

The scoping review will synthesise the characteristics of PR interventions that have been evaluated in LTC, the outcomes and QIs that have been used to evaluate the interventions, and tools or models used to determine eligibility for PR. The review may identify gaps in the literature regarding characteristics of PR interventions, the outcomes used to evaluate them, and tools to determine eligibility for services. The review will also be the first step in formally identifying what outcomes and QIs clinicians, policymakers and researchers can use to evaluate PR in LTC at the person, facility and system levels. The results of the scoping review will be disseminated via publication in health service journals and presentation at conferences specific to PR, LTC and the aging population. Although research ethics board approval will not be required for the scoping review, approval from the University of Waterloo research ethics board will be obtained for the formal consensus process and stakeholders participating in the nominal group technique will complete informed consent to participate. Methods and results of the consensus process will be published in a separate report. The scoping review will not only provide clinicians and policymakers with knowledge on how to evaluate the impact and quality of PR services in LTC, but will also identify the gaps in knowledge and identify areas for future research for PR in LTC.
Author affiliations

${ }^{1}$ Department of Kinesiology, University of Waterloo, Waterloo, Canada

${ }^{2}$ Department of Medicine, McMaster University, Hamilton, Canada

${ }^{3}$ University of Waterloo, Waterloo, Canada

${ }^{4}$ The Centre for Family Medicine, Kitchener, Canada

${ }^{5}$ Department of Family Medicine, McMaster University, Hamilton, Cananda

${ }^{6}$ CFFM-RIA Directorship for Mobility and Falls, Waterloo, Canada

${ }^{7}$ Department of Physical Therapy, University of Toronto, Toronto, Canada

${ }^{8}$ Toronto Rehabilitation Institute-University Health Network, Toronto, Canada

${ }^{9}$ Schelegel-UW Research Institute for Aging, Waterloo, Canada

Acknowledgements The authors would like to thank Rebecca Hutchinson and Jackie Stapleton, liaison librarians at the University of Waterloo, for assistance with the database search strategy. We gratefully acknowledge the opportunity to collaborate with Jaimie Killingbeck, Michael Sharratt and others from the Schlegel-UW Research Institute for Aging on this project.

Contributors $\mathrm{CM}$ was involved in writing the protocol. JG, AP, KB, JH and JM were involved in editing the protocol. LG was involved in project conception and editing the protocol.

Funding This work is funded by the Networks of Centres of Excellence: Technology Evaluation in the Elderly (TVN) Network Knowledge Synthesis Grant, KS2014-08. JH is the Ontario Home Care research and knowledge exchange chair funded by the Ontario Ministry of Health and Long-term Care. LG is the recipient of Canadian Institutes of Health Research (CIHR http:// www.cihr-irsc.gc.ca/e/193.html) New Investigator Award and an Early Researcher Award from the Ontario Ministry of Research and Innovation. $L G$ received funds from Merck Canada Inc., connected to the Early Researcher Award from the Ontario Ministry of Research and Innovation.

Competing interests None declared.

Provenance and peer review Not commissioned; externally peer reviewed.

Open Access This is an Open Access article distributed in accordance with the Creative Commons Attribution Non Commercial (CC BY-NC 4.0) license, which permits others to distribute, remix, adapt, build upon this work noncommercially, and license their derivative works on different terms, provided the original work is properly cited and the use is non-commercial. See: http:// creativecommons.org/licenses/by-nc/4.0/

\section{REFERENCES}

1. Living arrangements of seniors, Census in brief. 98-312-X2011003, 2011;1-8.

2. CLHIA. Report on long-term care policy. 2012;1-21.

3. Hirdes JP, Mitchell L, Maxwell CJ, et al. Beyond the 'iron lungs of gerontology': using evidence to shape the future of nursing homes in Canada. Can J Aging 2011;30:371-90.

4. Doupe $M$, St John $P$, Chateau $D$, et al. Profiling the multidimensional needs of new nursing home residents: evidence to support planning. J Am Med Dir Assoc 2012;13:487.e9-17.

5. Canadian Physiotherapy Association. Description of physiotherapy in Canada. 2012.

6. Crocker T, Forster A, Young J, et al. Physical rehabilitation for older people in long-term care. Cochrane Database Syst Rev 2013;2: CD004294.

7. Donabedian A. Evaluating the quality of medical care: part 2 Milbank Mem Fund Q 1966;11:166-206.

8. Norton PG, Murray M, Doupe MB, et al. Facility versus unit level reporting of quality indicators in nursing homes when performance monitoring is the goal. BMJ Open 2014;4:e004488.

9. Berg K, Mor V, Morris J, et al. Identification and evaluation of existing nursing homes quality indicators. Health Care Financ Rev 2002;23:19-36.

10. Jones RN, Hirdes JP, Poss JW, et al. Adjustment of nursing home quality indicators. BMC Health Serv Res 2010;10.

11. Health Quality Ontario. Long-term care public reporting website technical report table. http://www.hqontario.ca/public-reporting/ long-term-care/information-about-quality-indicators/ltc-technicalreport-table (accessed 20 Oct 2014).

12. Berg K, Sherwood S, Murphy K, et al. Rehabilitation in nursing homes: a cross-national comparison of recipients. Age Ageing 1997;26(Suppl. 2):37-42. 
13. Leemrijse CJ, De Boer ME, Van Den Ende CHM, et al. Factors associated with physiotherapy provision in a population of elderly nursing home residents; a cross sectional study. BMC Geriatr 2007;7.

14. Rolland Y, Pillard F, Klapouszczak A, et al. Exercise program for nursing home residents with Alzheimer's disease: a 1-year randomized, controlled trial. J Am Geriatr Soc 2007;55:158-65.

15. Roach KE, Tappen RM, Kirk-Sanchez N, et al. A randomized controlled trial of an activity specific exercise program for individuals with alzheimer disease in long-term care settings. J Geriatr Phys Ther 2011;34:50-6.

16. Christofoletti G, Oliani MM, Gobbi S, et al. A controlled clinical trial on the effects of motor intervention on balance and cognition in institutionalized elderly patients with dementia. Clin Rehabil 2008;22:618-26.

17. Ontario Ministry of Health and Long-Term Care. Policy for the Operation of Short-Stay Beds Under the Long-Term Care Homes Act. 2007.

18. Ontario Ministry of Health and Long-term Care. Frequently asked questions for physiotherapists. 2013. http://www.health.gov.on.ca/en/ pro/programs/physio/faq.aspx (accessed 19 Jun 2014).
19. Kochersberger G, Hielema F, Westlund R. Rehabilitation in the nursing home: how much, why, and with what results. Public Health Rep 1994;109:372-6.

20. Arksey H, O'Malley L. Scoping studies: towards a methodological framework. Int J Soc Res Methodol 2005;8:19-32.

21. Levac D, Colquhoun H, O'Brien KK. Scoping studies: advancing the methodology. Implement Sci 2010;5:69.

22. Health Canada. Long-term facilities-based care. http://www.hc-sc.gc.ca/ hcs-sss/home-domicile/longdur/index-eng.php (accessed 8 Dec 2014).

23. Mazurek Melnyk B, Fineout-Overholt E. Making the case for evidence-based practice and cultivating a spirit of inquiry. In: Fineout-Overholt E, ed. Evidence-based practice in nursing and health care: a guide to best practice. LWW, 2005:10.

24. Delbecq A, Van de Ven A, Gustafson D eds. Group techniques for program planning: a guide to nominal group and Delphi processes. Wisconsin: Green Briar Press, 1986.

25. Wainwright $D$, Boichat $C$, McCracken LM. Using the nominal group technique to engage people with chronic pain in health service development. Int $J$ Health Plann Manage 2014;29:52-69. 